\title{
Motion of isolated open vortex filaments evolving under the truncated local induction approximation
}

Robert A. Van Gorder

Citation: Physics of Fluids 29, 115105 (2017); doi: 10.1063/1.5005113

View online: https://doi.org/10.1063/1.5005113

View Table of Contents: http://aip.scitation.org/toc/phf/29/11

Published by the American Institute of Physics

\section{Articles you may be interested in}

Analysis of flapping motion of reattaching shear layer behind a two-dimensional backward-facing step

Physics of Fluids 29, 115104 (2017); 10.1063/1.4996622

Elliptical, parabolic, and hyperbolic exchanges of energy in drag reducing plane Couette flows

Physics of Fluids 29, 115106 (2017); 10.1063/1.5010047

From two-dimensional to three-dimensional turbulence through two-dimensional three-component flows Physics of Fluids 29, 111101 (2017); 10.1063/1.4990082

Universality of local dissipation scales in turbulent boundary layer flows with and without free-stream turbulence

Physics of Fluids 29, 115103 (2017); 10.1063/1.4996200

Attenuation of turbulence by the passive control of sweep events in a turbulent boundary layer using microcavities

Physics of Fluids 29, 115102 (2017); 10.1063/1.4995466

An overview of the statistical properties of two-dimensional turbulence in fluids with particles, conducting fluids, fluids with polymer additives, binary-fluid mixtures, and superfluids

Physics of Fluids 29, 111112 (2017); 10.1063/1.4986802

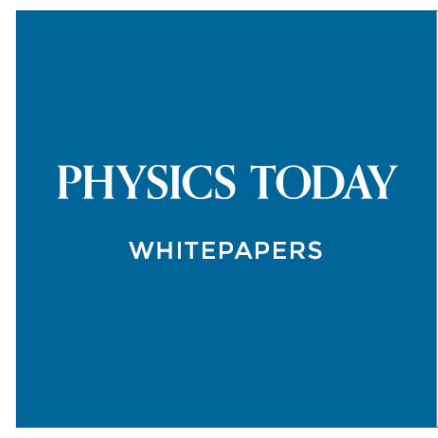

ADVANCED LIGHT CURE ADHESIVES

Take a closer look at what these environmentally friendly adhesive systems can do

\section{READ NOW}

PRESENTED BY

Q.MASTERBOND 


\title{
Motion of isolated open vortex filaments evolving under the truncated local induction approximation
}

\author{
Robert A. Van Gorder ${ }^{\text {a) }}$ \\ Mathematical Institute, University of Oxford, Andrew Wiles Building, Radcliffe Observatory Quarter, \\ Woodstock Road, Oxford OX2 6GG, United Kingdom
}

(Received 17 September 2017; accepted 24 October 2017; published online 10 November 2017)

\begin{abstract}
The study of nonlinear waves along open vortex filaments continues to be an area of active research. While the local induction approximation (LIA) is attractive due to locality compared with the nonlocal Biot-Savart formulation, it has been argued that LIA appears too simple to model some relevant features of Kelvin wave dynamics, such as Kelvin wave energy transfer. Such transfer of energy is not feasible under the LIA due to integrability, so in order to obtain a non-integrable model, a truncated LIA, which breaks the integrability of the classical LIA, has been proposed as a candidate model with which to study such dynamics. Recently Laurie et al. ["Interaction of Kelvin waves and nonlocality of energy transfer in superfluids," Phys. Rev. B 81, 104526 (2010)] derived truncated LIA systematically from Biot-Savart dynamics. The focus of the present paper is to study the dynamics of a section of common open vortex filaments under the truncated LIA dynamics. We obtain the analog of helical, planar, and more general filaments which rotate without a change in form in the classical LIA, demonstrating that while quantitative differences do exist, qualitatively such solutions still exist under the truncated LIA. Conversely, solitons and breather solutions found under the LIA should not be expected under the truncated LIA, as the existence of such solutions relies on the existence of an infinite number of conservation laws which is violated due to loss of integrability. On the other hand, similarity solutions under the truncated LIA can be quite different to their counterparts found for the classical LIA, as they must obey a $t^{1 / 3}$ type scaling rather than the $t^{1 / 2}$ type scaling commonly found in the LIA and Biot-Savart dynamics. This change in similarity scaling means that Kelvin waves are radiated at a slower rate from vortex kinks formed after reconnection events. The loss of soliton solutions and the difference in similarity scaling indicate that dynamics emergent under the truncated LIA can indeed differ a great deal from those previously studied under the classical LIA. Published by AIP Publishing. https://doi.org/10.1063/1.5005113
\end{abstract}

\section{INTRODUCTION}

The study of vortex filament dynamics in quantum fluids has been of great interest, motivated in large part due to the fact that quantized vortex filaments have been experimentally observed in superfluid helium. ${ }^{1}$ Due to the low viscosity of superfluid helium, such quantized vortex filaments can persist at larger time scales than their classical vortex filament counterparts, making such filament solutions realizable in experiments. Furthermore, vortex filament tangles and vortex reconnection events have been seen as harbingers of quantum turbulence, and indeed such vortex dynamics have been shown to degenerate into turbulence in a number of numerical simulations. ${ }^{1}$ Therefore, the construction of vortex filament solutions in the low temperature regime is of great interest physically as well as mathematically.

To begin with, recall that in the very low temperature regime, the self-induced dynamics of a thin vortex filament are given by the Biot-Savart formulation ${ }^{2,3}$

$$
\mathbf{r}_{t}=\frac{\hat{\Gamma}}{4 \pi} \int \frac{(\mathbf{s}-\mathbf{r}) \times d \mathbf{s}}{|\mathbf{s}-\mathbf{r}|^{3}}
$$

a) Author to whom correspondence should be addressed: Robert. VanGorder@maths.ox.ac.uk
Here $\hat{\Gamma}=2 \pi \hbar / m$ is the quantum velocity of circulation, $m$ is the particle mass, and the integration is performed over $|\mathbf{r}-\mathbf{s}|>a_{0}$ (where $a_{0}$ is the core radius). The integration is over the length of the filament $\mathbf{r}$. The non-local dynamics are often approximated by the local induction approximation (LIA), which gives ${ }^{4,5}$

$$
\mathbf{r}_{t}=\frac{\hat{\Gamma}}{4 \pi} \ln \left(\frac{\ell}{a_{0}}\right) \kappa \mathbf{t} \times \mathbf{n},
$$

where $\kappa$ is the curvature, $\mathbf{t}$ is the tangent vector to the curve, $\mathbf{n}$ is the normal vector to the curve, $\Gamma=\frac{\hat{\Gamma}}{4 \pi} \ln \left(\frac{\ell}{a_{0}}\right)$, and $\ell$ denotes the inter-vortex distance. This formulation introduces a cutoff at $a<|\mathbf{r}-\mathbf{s}|$ to avoid the singularity in (1). The well-known Hasimoto transformation ${ }^{6}$ can be used to map (2) directly into the cubic nonlinear Schrödinger (NLS) equation, with the resulting complex scalar potential encoding the curvature and torsion of the filament. This approach has been used in a number of studies, where one obtains a particular solution to the cubic NLS equation and inverts the Hasimoto map in order to obtain a vortex filament solution to the LIA. Solutions such as one-solitons, ${ }^{6} \mathrm{~N}$-solitons, ${ }^{7}$ breathers, ${ }^{8,9}$ multiple breathers, ${ }^{10}$ and torus knots ${ }^{11}$ have been found via such an approach. Some of these theoretical results were even demonstrated to be experimentally relevant. ${ }^{12-14}$ 
The dynamics of Kelvin waves along vortex filaments continues to be an area of active research. While the LIA formulation is attractive due to locality (hence it is simpler to solve analytically or numerically) compared with the nonlocal Biot-Savart formulation, ${ }^{15}$ it has been argued that the LIA appears too simple to describe Kelvin wave energy transfer. This is due to the fact that the LIA conserves an infinite number of integrals of motion (due to integrability), and wave resonances are absent in all orders, preventing the exchange of energy between Kelvin waves. To get around this, a truncated LIA, which breaks LIA integrability, was proposed. ${ }^{16}$ Later, a different but equivalent truncated LIA was derived. ${ }^{15}$ The benefit of the latter is that it was derived in a systematic way from the Biot-Savart dynamics, while the truncated LIA of Ref. 16 was obtained in a more ad hoc manner. To contrast the LIA (2) with the truncated LIA, we shall subsequently refer to (2) as the classical LIA.

In the present paper, we apply the types of analysis considered previously for the 2D-LIA and the Biot-Savart model to the so-called Local Nonlinear Equation (LNE) which was derived as a kind of truncated LIA. Despite recent interest in this model, this has not been a systematic study of nonlinear waves emergent on vortex filaments under the LNE. The primary goal of the present paper is to therefore identify qualitative differences and similarities between the structure of nonlinear waves along vortex filament solutions to the LNE and previously studied solutions to the 2D-LIA and Biot-Savart models. In Sec. II, we review the derivation of the LNE so as to put it in the context of other existing models. We review results on Kelvin waves along line filaments under the LNE, akin to what was found for the 2D-LIA, with an additional correction to the dispersion relation. We also apply the Painlevé test in order to prove that the LNE does indeed break the integrability of the LIA. In Sec. III, we demonstrate the existence of standing waves which correspond to planar vortex filaments, as well as more general standing waves (or, stationary states), comparing the results with general stationary states for the 2D-LIA and planar waves for the Biot-Savart model. Self-similar structures have recently been observed experimentally and exhibited analytically for both the 2D-LIA and the Biot-Savart dynamics, and while we demonstrate that self-similar structures can exist analytically in the LNE framework in Sec. IV, the self-similar scalings will be different from those of the LIA and Biot-Savart models, suggesting that any self-similar energy cascade would differ under the LNE dynamics. We summarize the results in Sec. V.

\section{THE LOCAL NONLINEAR EQUATION}

The Biot-Savart dynamics describing the motion of a thin, quantized vortex filament are given by (1). Svistunov ${ }^{2}$ studied vortex filament dynamics for a low-temperature superfluid in the Cartesian form, which allows one to immediately see the geometry of the line filaments. In contrast, the curvaturetorsion formulation (which is what is used to map the LIA into the cubic NLS equation) requires extra effort in order to recover the Cartesian solutions. In particular, Svistunov considered the Hamiltonian formulation of the Biot-Savart dynamics (1) in Cartesian coordinates. Writing the vortex filament curve in the form $\mathbf{r}(z, t)=(x(z, t), y(z, t), z)$ and introducing a complex scalar function $w(x, t)=x(z, t)+i y(z, t)$, Svistunov obtained the Hamiltonian system

$$
i w_{t}=\frac{\delta H[w]}{\delta w^{*}},
$$

where

$H[w]=\frac{\Gamma}{4 \pi} \int \frac{1+\operatorname{Re}\left(w_{x}^{*}\left(z_{1}, t\right) w_{x}\left(z_{2}, t\right)\right)}{\sqrt{\left(z_{1}-z_{2}\right)^{2}+\left|w\left(z_{1}, t\right)-w\left(z_{2}, t\right)\right|^{2}}} d z_{1} d z_{2}$

and the star denotes complex conjugation. Under the assumption that the vortex filament $\mathbf{r}$ is of sufficient bounded variation, that is,

$$
\frac{\left|w\left(z_{1}, t\right)-w\left(z_{2}, t\right)\right|}{\left|z_{1}-z_{2}\right|} \ll 1,
$$

one can apply the LIA procedure to Hamiltonian (4), obtaining the local approximation

$$
\hat{H}[w]=2 \frac{\Gamma}{4 \pi} \Lambda_{0} \int \sqrt{1+\left|w_{z}(z, t)\right|^{2}} d z .
$$

To get this approximation, one expands in a double limit where $\left|w_{z}\right| \ll 1$ (bounded variation assumption) and $\Lambda_{0} \gg 1$ where $\Lambda_{0}=\ln \left(\frac{\ell}{a}\right)$ and $\ell$ is a reference length. Note that we have selected the reference axis to correspond to the $z$ coordinate, although this choice is arbitrary. Then, the equation of motion from this LIA Hamiltonian gives us

$$
i w_{t}+\left(\frac{w_{z}}{\sqrt{1+\left|w_{z}\right|^{2}}}\right)=0,
$$

and we shall refer to this as the 2D-LIA after the paper in which it was derived. ${ }^{16}$ This equation has been studied in a number of contexts, and once any such solution is found, we directly have a vortex filament solution of the form $\mathbf{r}=(\operatorname{Re}(w(z, t))$, $\operatorname{Im}(w(z, t)), z)$.

As pointed out by Ref. 15, there is a clear need for a simpler model for nonlinear Kelvin waves than the full Biot-Savart dynamics. Yet, this model should be more complicated than the LIA (which is really the first approximation in $\Lambda_{0}^{-1}$ ), in order to describe the energy transfer over turbulent scales. Motivated by this need, an ad hoc model was introduced in Ref. 16 which has the simplest form with all the scaling properties and solutions of the original Biot-Savart model preserved. This model was called the truncated LIA (TLIA), and the corresponding Hamiltonian is given by

$$
H^{\mathrm{TLIA}}[w]=\frac{\Gamma}{4 \pi} \Lambda_{0} \int\left\{\left|w_{z}\right|^{2}-\frac{1}{4}\left|w_{z}\right|^{4}\right\} d z .
$$

The name TLIA arises from the fact that it can formally be obtained by expanding the LIA Hamiltonian in powers of the modulus $\left|w_{z}\right|^{2}$ and then truncating at the fourth order. This truncation leads to the breaking of the LIA integrability although it is claimed to preserve all the important scalings. The utility of this model was demonstrated in Ref. 16.

Later, Ref. 15 showed that it is possible to obtain a type of truncated approximation of the Biot-Savart Hamiltonian in a more systematic way. This Hamiltonian was given by

$$
H^{\mathrm{LNE}}[\tilde{w}]=\frac{\Gamma}{4 \pi} \int\left\{\Lambda\left|\tilde{w}_{z}\right|^{2}-\frac{1}{12}\left|\tilde{w}_{z}\right|^{6}\right\} d z,
$$


where $\Lambda=\Lambda_{0}-\gamma-\frac{3}{2} \approx \Lambda_{0}-2, \gamma \approx 0.5772$ is the Euler constant, and $\tilde{w}(z, t)=w(z, t)+O\left(\left|w_{z}\right|^{3}\right)$. Therefore, when $w(z, t)$ is of sufficient bounded variation in coordinate $z$, i.e., $\left|w_{z}\right|^{2} \ll 1$, we have $\tilde{w}(z, t) \approx w(z, t)$. The replacement $\Lambda_{0} \rightarrow$ $\Lambda$ is equivalent to the replacement of the core radius with an effective core radius, $a_{0} \rightarrow a=a_{0} \exp (\Gamma+3 / 2) \approx 8 a_{0}$ with $\Lambda=\ln (\ell / a)$. This Hamiltonian has the equation of motion,

$$
i \frac{\partial \tilde{w}}{\partial t}+\frac{\Gamma}{4 \pi} \frac{\partial}{\partial z}\left[\left(\Lambda-\frac{1}{4}\left|\frac{\partial \tilde{w}}{\partial z}\right|^{4}\right) \frac{\partial \tilde{w}}{\partial z}\right]=0,
$$

which is therefore a local equation approximating the motion of a thin vortex filament, under the assumptions outlined earlier. Equation (10) is referred to as the local nonlinear equation (LNE) in Ref. 15, and to distinguish it from the LIA, 2DLIA, and Biot-Savart formulations, we shall refer to it as such throughout this paper. Various wave-wave interactions under this model were previously explored. ${ }^{15}$

\section{A. Traveling waves along vortex filaments under the LNE}

A common solution to consider is the helical filament configuration. Such configurations model the propagation of Kelvin waves along line filaments in superfluid helium, and the motion of such configurations in local models is usually determined by a simple dispersion relation. For the Biot-Savart formulation, the "cut-off" method has been used to the study of helical vortex filaments. ${ }^{19-22}$ Analytical results are also common under the LIA, ${ }^{23-25}$ which is much simpler to solve than the Biot-Savart model in the case of helical vortex filaments. Recently the LIA was used to regularise the core region of a helical filament (rather than using the cut-off method) while the Biot-Savart integral "tails" were retained, ${ }^{26}$ and these analytical results were in good general agreement with the aforementioned cut-off method results, while also making direct comparison with the LIA results more feasible.

To begin, assume a plane wave solution of the form

$$
\tilde{w}(z, t)=A \exp \left(i\left\{k\left(z-z_{0}\right)-\omega t\right\}\right) .
$$

Placing (11) into (10), we find the dispersion relation

$$
\omega=\frac{\Gamma}{4 \pi} k^{2}\left(\Lambda-\frac{A^{4} k^{4}}{4}\right) .
$$

We therefore find

$$
\tilde{w}(z, t)=A \exp \left(i\left\{k\left(z-z_{0}\right)-\frac{\Gamma}{4 \pi} k^{2}\left(\Lambda-\frac{A^{4} k^{4}}{4}\right) t\right\}\right),
$$

hence the helical filament takes the form

$$
\mathbf{r}(z, t)=\left[\begin{array}{c}
A \cos \left(k\left(z-z_{0}\right)-\frac{\Gamma}{4 \pi} k^{2}\left(\Lambda-\frac{A^{4} k^{4}}{4}\right) t\right) \\
A \sin \left(k\left(z-z_{0}\right)-\frac{\Gamma}{4 \pi} k^{2}\left(\Lambda-\frac{A^{4} k^{4}}{4}\right) t\right) \\
z
\end{array}\right] .
$$

As done in Sec. III of Ref. 17, one may similarly show that for the LNE, the only pure traveling wave solutions are helical filaments.

\section{B. Loss of integrability in the LNE}

Solitons are possible in the LIA and have been explored in the Cartesian 2D-LIA, as well. However, such solutions rely on integrability of the underlying partial differential equation (PDE). While the LNE is claimed to break the integrability of the LIA due to the truncation taken, ${ }^{15}$ it is not immediately clear that it is not integrable or conditionally integrable in some special cases which may then be exploited to recover solitons or other exact solutions. To determine whether the LNE is conditionally integrable, we apply the Painlevé test (for details of the method, see Refs. 27-29; for some recent applications, see Refs. 30 and 31). Consider an expansion of $\tilde{w}$ on a singular manifold,

$$
\tilde{w}=\frac{W_{0}}{\varphi(z, t)^{g}}
$$

where $g$ and $W_{0}$ are constants. If $g$ is a positive integer, and resonance conditions can be accounted for (so that the dominant balance induces a terminating Laurent series in the singular manifold), then the resulting equation is integrable (or, conditionally integrable, if the result depends on system parameters). The dominant balance is found matching

$$
\frac{\partial \tilde{w}}{\partial t} \sim \frac{\partial}{\partial z}\left[\left|\frac{\partial \tilde{w}}{\partial z}\right|^{4} \frac{\partial \tilde{w}}{\partial z}\right]
$$

and this yields $\varphi^{-(g+1)}=\varphi^{-(5 g+6)}$. The dominant balance then implies that $g=-5 / 4$, rather than a positive integer, and thus, the LNE indeed fails the Painlevé test. As claimed in earlier studies, the LNE destroys the particular integrability of the LIA and, as we show here, is further neither integrable nor conditionally integrable at all. Hence, one does not expect soliton or related solutions (such as breathers) — the existence of which relies on having a sufficient number of conservation laws.

\section{VORTEX FILAMENTS MOVING WITHOUT CHANGE IN GEOMETRY}

A helical filament is but one example of a vortex filament which moves without a change in form. Another exact solution to the LIA is the so-called planar vortex filament, as it maintains its form while lying on a plane that rotates with time along the central axis of the filament. The planar solution was discovered by Da Rios. ${ }^{4,32}$ The solution was later rediscovered by Hasimoto ${ }^{33}$, who related it to elastica. $\mathrm{Kida}^{24}$ compared the planar filament with other types of solutions and later considered a numerical stability analysis of the solution. ${ }^{36}$ A direct derivation in Cartesian coordinates was given in Ref. 34, and a perturbation solution (using strained coordinates and parameters) was presented in Ref. 35. An analytical stability analysis for the orbital stability (spectral stability) was also obtained. ${ }^{37}$ It is also possible to formulate the LIA in terms of the unknown tangent vector as a function of arc length and time, as done in Ref. 8. A planar solution in this framework was given by Ref. 39.

Relating to more complicated physics, Ref. 38 studied the influence of background flows on planar filaments. The planar vortex filament solution was also recently shown to exist $^{40}$ for the non-local Biot-Savart dynamics, which is more complicated yet more realistic formulation for the motion of classical vortex filaments. Analytical perturbation solutions were also constructed in Ref. 40 for the Biot-Savart case. 


\section{A. Planar filaments}

In order to study planar filaments in the LNE, let us first introduce the standing wave ansatz

$$
\tilde{w}(z, t)=\phi(z) \exp \left(-i \frac{\Gamma \Lambda}{4 \pi} \omega t\right) .
$$

The transformation (17) puts Eq. (10) into the form of an ordinary differential equation for $\phi$,

$$
\omega \phi+\frac{d^{2} \phi}{d z^{2}}-\frac{\epsilon}{5} \frac{d}{d z}\left(\frac{d \phi}{d z}\right)^{5}=0 .
$$

Here,

$$
\epsilon=\frac{5}{4 \Lambda} \ll 1
$$

is a small parameter, as we recall that $\Lambda \gg 1$. Writing (18) in the form

$$
\omega \phi+\left(1-\epsilon\left(\frac{d \phi}{d z}\right)^{4}\right) \frac{d^{2} \phi}{d z^{2}}=0,
$$

we see that there exists a first integral

$$
\omega \phi^{2}+\left(\frac{d \phi}{d z}\right)^{2}-\frac{\epsilon}{3}\left(\frac{d \phi}{d z}\right)^{6}=I_{0},
$$

where $I_{0}$ is an integration constant. From the form of this first integral, we should have that the spectral parameter $\omega$ satisfies $\omega>0$ in order to obtain bounded and periodic solutions. In order to study such solutions, it is sufficient to pick boundary data of the form $\phi(0)=\phi_{\max }$ and $\phi^{\prime}(0)=0$. This, in turn, implies that $I_{0}=\omega \phi_{\max }^{2}$, i.e.,

$$
\omega \phi^{2}+\left(\frac{d \phi}{d z}\right)^{2}-\frac{\epsilon}{3}\left(\frac{d \phi}{d z}\right)^{6}=\omega \phi_{\max }^{2} .
$$

Now, as $\epsilon \rightarrow 0$ in (22), we obtain the exact solution $\phi(z)=$ $\phi_{\max } \cos (\sqrt{\omega} z)$. So, for small $\epsilon$ (which is again consistent with the property of the LNE that $\Lambda \gg 1$ ), we expect to obtain periodic orbits that are essentially perturbations of this solution.

Employing a strained parameter approach to construct a perturbation solution which keeps terms up to $\epsilon^{2}$, we obtain a solution of the form

$$
\phi(z)=\phi_{\max } \cos (Z)+\epsilon \phi_{1}(Z)+\epsilon^{2} \phi_{2}(Z)+O\left(\epsilon^{3}\right),
$$

where

$$
Z=\sqrt{\omega}\left(1+\epsilon Z_{1}+\epsilon^{2} Z_{2}+O\left(\epsilon^{3}\right)\right) z
$$

Selecting the terms $Z_{1}$ and $Z_{2}$ to remove secular growth in the perturbation expansion, we find that

$$
Z_{1}=\frac{1}{16} \omega^{2} \phi_{\max }^{4} \quad \text { and } \quad Z_{2}=\frac{1}{64} \omega^{4} \phi_{\max }^{8}
$$

while we find that the first two perturbation corrections are given by

$\phi_{1}(Z)=\frac{1}{48} \omega^{2} \phi_{\max }^{5} \hat{\phi}_{1}(Z)$ and $\quad \phi_{2}(Z)=\frac{1}{64} \omega^{4} \phi_{\max }^{9} \hat{\phi}_{2}(Z)$,

where

$$
\begin{gathered}
\hat{\phi}_{1}(Z)=\cos (Z)-\frac{9}{8} \cos (3 Z)+\frac{1}{8} \cos (5 Z) \\
\hat{\phi}_{2}(Z)=\frac{2135}{2304} \cos (Z)-\frac{39}{32} \cos (3 Z)+\frac{103}{288} \cos (5 Z) \\
-\frac{329}{4608} \cos (7 Z)+\frac{3}{512} \cos (9 Z)
\end{gathered}
$$

Note that the period of such a planar solution is then approximated by

$$
\begin{aligned}
\tau & =\frac{2 \pi}{\sqrt{\omega}\left(1+\frac{1}{16} \omega^{2} \phi_{\max }^{4} \epsilon+\frac{39}{1024} \omega^{4} \phi_{\max }^{8} \epsilon^{2}\right)}+O\left(\epsilon^{3}\right) \\
& =\frac{2 \pi}{\sqrt{\omega}\left(1+\frac{5 \omega^{2} \phi_{\max }^{4}}{16 \Lambda}+\frac{975 \omega^{4} \phi_{\max }^{8}}{16384 \Lambda^{2}}\right)}+O\left(\Lambda^{-3}\right)
\end{aligned}
$$

With this, we can write

$$
\begin{aligned}
\phi(z)= & \phi_{\max } \cos \left(\frac{2 \pi z}{\tau}\right)+\frac{5}{192} \frac{\omega^{2} \phi_{\max }^{5}}{\Lambda} \hat{\phi}_{1}\left(\frac{2 \pi z}{\tau}\right) \\
& +\frac{25}{1024} \frac{\omega^{4} \phi_{\max }^{9}}{\Lambda^{2}} \hat{\phi}_{2}\left(\frac{2 \pi z}{\tau}\right)+O\left(\Lambda^{-3}\right) .
\end{aligned}
$$

The planar vortex filament under the LNE is then given [up to $O\left(\Lambda^{-2}\right)$ ] by

$$
\mathbf{r}(z, t)=\left[\begin{array}{c}
\left\{\phi_{\max } \cos \left(\frac{2 \pi z}{\tau}\right)+\frac{5}{192} \frac{\omega^{2} \phi_{\max }^{5}}{\Lambda} \hat{\phi}_{1}\left(\frac{2 \pi z}{\tau}\right)+\frac{25}{1024} \frac{\omega^{4} \phi_{\max }^{9}}{\Lambda^{2}} \hat{\phi}_{2}\left(\frac{2 \pi z}{\tau}\right)\right\} \cos \left(\frac{\Gamma \Lambda}{4 \pi} \omega t\right) \\
-\left\{\phi_{\max } \cos \left(\frac{2 \pi z}{\tau}\right)+\frac{5}{192} \frac{\omega^{2} \phi_{\max }^{5}}{\Lambda} \hat{\phi}_{1}\left(\frac{2 \pi z}{\tau}\right)+\frac{25}{1024} \frac{\omega^{4} \phi_{\max }^{9}}{\Lambda^{2}} \hat{\phi}_{2}\left(\frac{2 \pi z}{\tau}\right)\right\} \sin \left(\frac{\Gamma \Lambda}{4 \pi} \omega t\right)
\end{array}\right]+O\left(\Lambda^{-3}\right) .
$$

Both LIA and Biot-Savart results from the literature have an $O\left(\phi_{\max }^{2}\right)$ correction rate to both the amplitude and spatial periods of a planar filament solution, while the LNE solution has an $O\left(\phi_{\max }^{4}\right)$ correction rate. The corresponding formula for the spatial period of the LIA solution is given in Eq. (45) of Ref. 40 and for the Biot-Savart solution in Eq. (44) of Ref. 40.

\section{B. General filaments moving without change of form}

It is possible to generalize such planar solutions to obtain general rotating and translating waves along vortex filaments. Purely rotating solutions that generalize the planar solutions were considered in Ref. 41, and such solutions were recently 
studied for the 2D-LIA in Ref. 17 where solutions are permitted to rotate and translate within the spatial domain. Related stationary solutions were also obtained for the Wadati-KonnoIchikawa-Shimizu (WKIS) equations and then mapped into the 2D-LIA in Ref. 18. The unifying theme behind all of these stationary solutions is that they can be used to model filaments which maintain their geometric form while rotating and translating in space.

Consider a stationary solution ansatz

$$
\tilde{w}(z, t)=(4 \Lambda)^{1 / 4} \exp \left(-\frac{i \Gamma \Lambda}{4 \pi} \omega t\right) \Phi(z)
$$

for $\omega>0$, which puts (10) into the form

$$
\omega \Phi+\frac{d}{d z}\left\{\left(1-\left|\frac{d \Phi}{d z}\right|^{4}\right) \frac{d \Phi}{d z}\right\}=0 .
$$

Defining $\Psi=\frac{d \Phi}{d z}$ and differentiating (33), we obtain

$$
\omega \Psi+\frac{d^{2}}{d z^{2}}\left\{\left(1-|\Psi|^{4}\right) \Psi\right\}=0 .
$$

Next, writing $\Psi(z)=R(z) \exp (i \theta(z))$ and placing this into (34), and then separating into real and imaginary parts, we obtain

$$
\begin{array}{r}
\omega R+\frac{d^{2}}{d z^{2}}\left\{\left(1-R^{4}\right) R\right\}-\left(1-R^{4}\right) R\left(\frac{d \theta}{d z}\right)^{2}=0, \\
\left(1-R^{4}\right) R \frac{d^{2} \theta}{d z^{2}}+2 \frac{d \theta}{d z} \frac{d}{d z}\left\{\left(1-R^{4}\right) R\right\}=0 .
\end{array}
$$

Solving (36) gives

$$
\frac{d \theta}{d z}=C\left(1-R^{4}\right)^{-2} R^{-2}
$$

where $C$ is a constant, and one more integration gives the phase

$$
\theta(z)=\theta(0)+C \int_{0}^{z}\left(1-R(\hat{z})^{4}\right)^{-2} R(\hat{z})^{-2} d \hat{z} .
$$

Placing (37) into (35), we obtain a single equation for $R$, namely,

$$
\omega R+\frac{d^{2}}{d z^{2}}\left\{\left(1-R^{4}\right) R\right\}-C^{2}\left(1-R^{4}\right)^{-3} R^{-3}=0,
$$

and, upon rearranging, we find

$$
\left(1-5 R^{4}\right) \frac{d^{2} R}{d z^{2}}-20 R^{3}\left(\frac{d R}{d z}\right)^{2}+\omega R-C^{2}\left(1-R^{4}\right)^{-3} R^{-3}=0
$$

Equation (40) defines a kind of nonlinear oscillator equation. Bounded periodic solutions can be shown to exist only if $1-5 R^{4} \neq 0$, i.e., $R \neq R^{*}=5^{-1 / 4} \approx 0.66874$, otherwise the solution will become singular at some finite $z$. Furthermore, we must have $\omega-C^{2}\left(1-R^{4}\right)^{-3} R^{-4}>0$, otherwise the solution will grow without bound. To demonstrate the existence of such periodic solutions in space, we give phase portraits in Fig. 1 for two distinct values of $C$.

Assuming the existence conditions on the amplitude and on $C$ are satisfied, there then exists a periodic solution $R(z)$. Placing the solution $R(z)$ back into the expressions for $\theta(z)$ and $\Psi(z)$, one then recovers $\Phi(z)$. Using the resulting expression in (32), we have

$$
\tilde{w}(z, t)=(4 \Lambda)^{1 / 4} \int^{z} R(\hat{z}) \exp \left(i\left\{\theta(z)-\frac{\Gamma \Lambda}{4 \pi} \omega t\right\}\right) d \hat{z} .
$$

From here, the general form of a vortex filament which moves without a change in form is given by

$$
\mathbf{r}(z, t)=\left[\begin{array}{c}
(4 \Lambda)^{1 / 4} \int^{z} R(\hat{z}) \cos \left(C \int^{\hat{z}}\left(1-R(q)^{4}\right)^{-2} R(q)^{-2} d q-\frac{\Gamma \Lambda}{4 \pi} \omega t\right) d \hat{z} \\
(4 \Lambda)^{1 / 4} \int^{z} R(\hat{z}) \sin \left(C \int^{\hat{z}}\left(1-R(q)^{4}\right)^{-2} R(q)^{-2} d q-\frac{\Gamma \Lambda}{4 \pi} \omega t\right) d \hat{z} \\
z
\end{array}\right] .
$$

From these general solutions, one can obtain a variety of geometric structures, akin to those general vortex filaments moving without a change in form shown in Refs. 17, 18, and 41 , and we shall not reproduce them here. We conclude that like in the case of the LIA or 2D-LIA, general solutions which maintain their form (i.e., stationary solutions) carry over to the LNE although the quantitative shape of solutions will naturally differ based on specific parameter selections.

\section{SELF-SIMILAR VORTEX FILAMENT STRUCTURES}

Self-similar vortex filament solutions can model the radiation of Kelvin waves from sharp kinks in hydrodynamic vortex filaments after reconnection events and can also model the development of singularities. ${ }^{47}$ Self-similar solutions to the LIA have been studied in relation to the dynamics of quantized vortex filaments in superfluid helium, ${ }^{42-46}$ and such dynamics are useful in the study of vortex collapse and turbulence. ${ }^{50-55}$

Numerical simulations have suggested that vortex tangles may exhibit a degree of self-similarity at small time scales, ${ }^{48}$ and self-similar structures localised in time have been observed in collapsing vortex rings just prior to core overlapping. ${ }^{49}$ Recent numerical evidence for self-similarity being maintained in the non-local Biot-Savart formulation was provided in Ref. 56, and recently self-similar solutions have shown to exist for the non-local Biot-Savart dynamics of an isolated vortex filament. ${ }^{57}$ A perturbation approximation to the self-similar vortex filament solution was also provided in Ref. 57. 


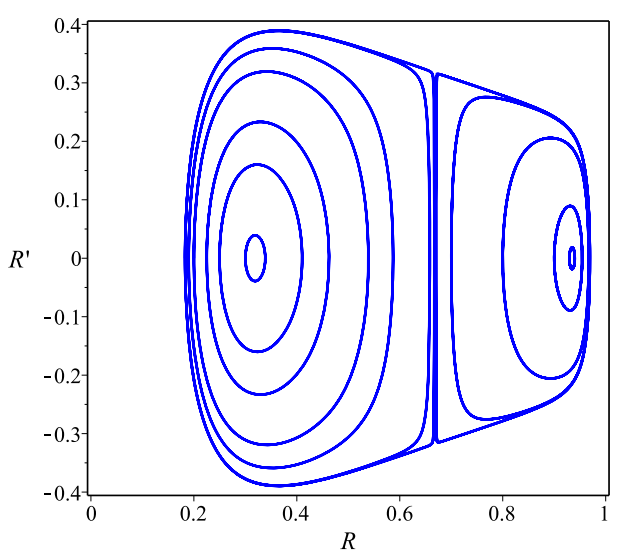

(a)

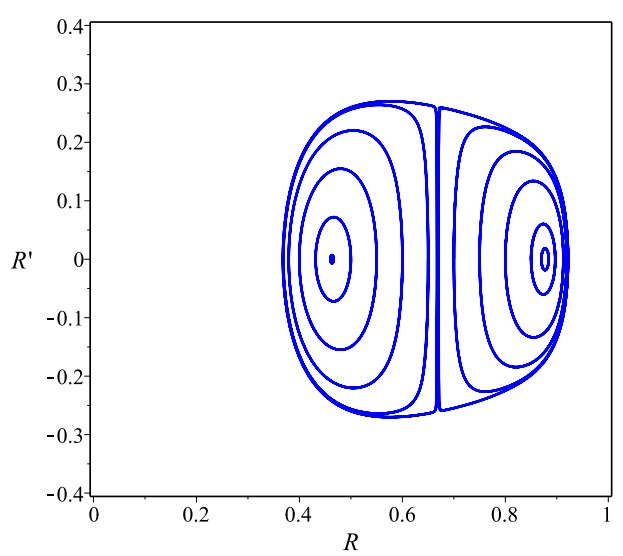

(b)
FIG. 1. Phase portraits for solutions of (40) when (a) $C=0.1$ and (b) $C=0.2$; we fix $\omega=1$. As $C$ increases, the existence region (based on the amplitude of $R$ ) sharply decreases. When $C=0.1$, we have that solutions exist for $0.183<R$ $<R^{*}$ and $R^{*}<R<0.969$, where $R^{*}=$ $5^{-1 / 4}$. When $C=0.2$, solutions exist for $0.368<R<R^{*}$ and $R^{*}<R<0.922$.
Since Eq. (10) is of a similar form to a derivative nonlinear Schrödinger equation, it makes sense to look for a similarity solution that takes the form $w(z, t) \sim t^{\alpha_{1}} f\left(z t^{\alpha_{2}}\right)$. Upon plugging this form into (10), we find that $\alpha_{1}=\frac{1}{3}$ and $\alpha_{2}=-\frac{1}{3}$ are the only parameter combination which gives a similarity solution. Therefore, let us more precisely consider a solution of the form

$$
\tilde{w}(z, t)=\left(\frac{4 \pi}{3 \Gamma \Lambda} t\right)^{1 / 3} f(\eta), \quad \text { where } \quad \eta=\left(\frac{3 \Gamma \Lambda}{4 \pi}\right)^{1 / 3} \frac{z}{t^{1 / 3}} .
$$

With this transformation, Eq. (10) is put into the form of the complex ordinary differential equation

$$
i\left(f-\eta \frac{d f}{d \eta}\right)+\frac{d^{2} f}{d \eta^{2}}-\frac{1}{4 \Lambda} \frac{d}{d \eta}\left(\left|\frac{d f}{d \eta}\right|^{4} \frac{d f}{d \eta}\right)=0 .
$$

Since $\Lambda \gg 1$, we can approximate (44) like

$$
i\left(\hat{f}-\eta \frac{d \hat{f}}{d \eta}\right)+\frac{d^{2} \hat{f}}{d \eta^{2}}=0
$$

up to zeroth order in $\Lambda^{-1}$. That is, $f(\eta)=\hat{f}(\eta)+O\left(\Lambda^{-1}\right)$. There exist two linearly independent solutions to this equation, one of which is $\hat{f}_{1}(\eta)=\eta$. To find the other solution, we set $\hat{f}_{2}(\eta)=\hat{f}_{1}(\eta) g(\eta)=\eta g(\eta)$, which gives

$$
\eta \frac{d^{2} g}{d \eta^{2}}+\left(2-i \eta^{2}\right) \frac{d g}{d \eta}=0
$$

One finds

$$
\begin{aligned}
g(\eta)= & -\frac{1}{\eta} \cos \left(\frac{\eta^{2}}{2}\right)-\sqrt{\pi} \mathrm{S}\left(\frac{\eta}{\sqrt{\pi}}\right) \\
& +i\left\{\sqrt{\pi} \mathrm{C}\left(\frac{\eta}{\sqrt{\pi}}\right)-\frac{1}{\eta} \sin \left(\frac{\eta^{2}}{2}\right)\right\},
\end{aligned}
$$

where $\mathrm{S}$ and $\mathrm{C}$ denote the Fresnel $\mathrm{S}$ and Fresnel $\mathrm{C}$ functions, respectively. Now, we can express

$$
\hat{f}(\eta)=\left(c_{1 R}+c_{1 I} i\right) \eta+\left(c_{2 R}+c_{2 I} i\right) \eta g(\eta),
$$

where $c_{1 R}, c_{1 I}, c_{2 R}$, and $c_{2 I}$ are real-valued constants. If we try to find a bounded solution, we obtain only the zero solution. However, recall that we really need $w_{z}$ to be sufficiently bounded. Therefore, we shall pick the arbitrary constants so that the derivative $\frac{d \hat{f}}{d \eta}$ is sufficiently small. The function $\hat{f}_{2}(\eta)=\eta g(\eta)$ gives oscillations around a linear trend, while the function $\hat{f}_{1}(\eta)=\eta$ simply has an additional linear trend. Picking $c_{1 R}=c_{1 I}=c_{2 I}=0$ and $c_{2 R}=\frac{\delta}{\sqrt{\pi}}$ for some small parameter $0<\delta<1$, we find that

$$
\begin{aligned}
\hat{f}(\eta)= & -\frac{\delta}{\sqrt{\pi}} \cos \left(\frac{\eta^{2}}{2}\right)-\delta \eta \mathrm{S}\left(\frac{\eta}{\sqrt{\pi}}\right) \\
& +i \delta \eta \mathrm{C}\left(\frac{\eta}{\sqrt{\pi}}\right)-i \frac{\delta}{\sqrt{\pi}} \sin \left(\frac{\eta^{2}}{2}\right) .
\end{aligned}
$$

We then have

$$
\frac{d \hat{f}}{d \eta}=-\delta \mathrm{S}\left(\frac{\eta}{\sqrt{\pi}}\right)+i \delta \mathrm{C}\left(\frac{\eta}{\sqrt{\pi}}\right) .
$$

Now,

$$
\max _{\eta \in \mathbb{R}} \sqrt{\left\{\mathrm{S}\left(\frac{\eta}{\sqrt{\pi}}\right)\right\}^{2}+\left\{\mathrm{C}\left(\frac{\eta}{\sqrt{\pi}}\right)\right\}^{2}} \approx 0.949<1,
$$

hence we have that

$$
\left|\frac{d \hat{f}}{d \eta}\right|=\delta \sqrt{\left\{\mathrm{S}\left(\frac{\eta}{\sqrt{\pi}}\right)\right\}^{2}+\left\{\mathrm{C}\left(\frac{\eta}{\sqrt{\pi}}\right)\right\}^{2}}<\delta
$$

for all $\eta \in \mathbb{R}$. Therefore, this approximate solution is of the desired bounded variation since we can control the first derivative to be within a modulus less than a prescribed small parameter $\delta$. Since

$$
\frac{\partial \tilde{w}}{\partial z}=\frac{d f}{d \eta}
$$

we have that

$$
\left|\frac{\partial \tilde{w}}{\partial z}\right|<\delta
$$

for all $z \in \mathbb{R}$ and all $t>0$, as well. This means that the solution remains of sufficiently small bounded variation to be physically meaningful, despite growing at a linear rate away from the origin (as this linear rate of growth is small enough). Therefore, we have the solution 


$$
\mathbf{r}(z, t)=\left[\begin{array}{c}
-\frac{\delta}{\sqrt{\pi}}\left\{\left(\frac{4 t}{3 \Gamma \Lambda}\right)^{1 / 3} \cos \left(\left(\frac{3 \Gamma \Lambda}{4 \pi}\right)^{2 / 3} \frac{z^{2}}{2 t^{2 / 3}}\right)+\sqrt{\pi} z \mathrm{~S}\left(\left(\frac{3 \Gamma \Lambda}{4 \pi}\right)^{1 / 3} \frac{z}{\sqrt{\pi} t^{1 / 3}}\right)\right\} \\
\frac{\delta}{\sqrt{\pi}}\left\{\sqrt{\pi} z \mathrm{C}\left(\left(\frac{3 \Gamma \Lambda}{4 \pi}\right)^{1 / 3} \frac{z}{\sqrt{\pi} t^{1 / 3}}\right)-\left(\frac{4 t}{3 \Gamma \Lambda}\right)^{1 / 3} \sin \left(\left(\frac{3 \Gamma \Lambda}{4 \pi}\right)^{2 / 3} \frac{z^{2}}{2 t^{2 / 3}}\right)\right\} \\
z
\end{array}\right]+O\left(\delta^{4}\right)
$$

which obeys the requisite boundedness condition for small- $\delta$ amplitude oscillations. Note that, since $\delta$ is small and $\Lambda$ is large, any corrections due to the nonlinear term in (44) will be of order $\delta^{4} \Lambda^{-1}$. Indeed, such contributions are very small and can be neglected. We provide a plot of solution (55) in Fig. 2.

While the nonlinearity was omitted when calculating the approximate solution, note that the influence of the nonlinearity does show up in the particular type of similarity solution $\left(t^{1 / 3}\right)$ obtained. One may compare this result with the $t^{1 / 2}$ similarity scaling obtained for the 2D-LIA directly ${ }^{17}$ or for the transformed solutions governed by the first equation in the WKIS hierarchy. ${ }^{18}$ The $t^{1 / 2}$ type scalings also appear in numerical studies for the LIA or Biot-Savart model, in addition to the fully non-local Biot-Savart asymptotics which were recently studied in Ref. 57; see, for instance, Eqs. (3.18) and (3.21) of Ref. 57. Thus, the appearance of a $t^{2 / 3}$ type similarity scaling is perhaps one of the greatest qualitative differences between the LNE and the LIA and Biot-Savart results.
It is worth pointing out that self-similar solutions are not simply of mathematical or theoretical interest but also have been observed in experiments. ${ }^{58}$ Indeed, in Ref. 58, solutions to the classical LIA with $t^{1 / 2}$ type scaling were shown to agree well with experimental observations near the core region (the kinked or $V$-shaped section of the filament). Linear approximations and dimensional scalings are often used to support a $t^{1 / 2}$ type scaling, see, for instance, Ref. 59. Still, there may be scenarios where a different kind of scaling, such as the $t^{1 / 3}$ scaling obtained for the local nonlinear equation, may be useful. Rorai et al. ${ }^{60}$ recently discussed that for certain initial configurations (such as initially orthogonal vortex lines), a $t^{1 / 3}$ type scaling separation before reconnection of vortex lines, followed by a $t^{2 / 3}$ scaling after reconnection, rather than the common $t^{1 / 2}$ type scaling, can be observed. Therefore, the $t^{1 / 3}$ type scalings obtained here may still be of physical relevance, for certain vortex filament configurations.
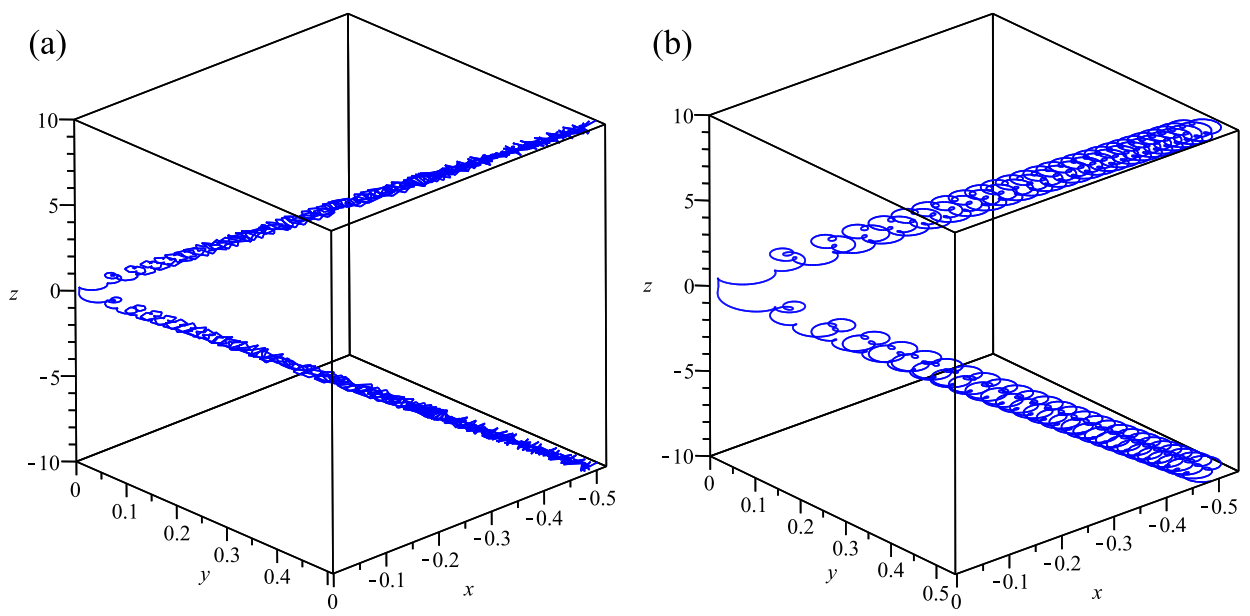

FIG. 2. Plot of the self-similar solution (55) to the LNE. Parameter values are taken to be $\delta=0.1, \Lambda=10, \Gamma=1$. In each panel, we plot the solution in space at time (a) $t=0.01$, (b) $t=0.1$, (c) $t=1$. As time increases, the kinked solution becomes more smooth, with the amplitude of radiated Kelvin waves increasing. Note that the curves never self-intersect, although the angle may make it appear that way.

(c)






\section{CONCLUSIONS}

We have considered several solutions to the local nonlinear equation derived in Ref. 15 from a truncated local induction approximation. Helical, planar, and, more generally, those filaments which move through space without a change in form are shown to exist under the local nonlinear equation, with primarily quantitative rather than qualitative differences appearing between the classical and truncated LIA solutions. On the other hand, certain solutions corresponding to coherent nonlinear structures along vortex linear, such as solitons and breather solutions, previously found under the classical LIA are not to be found under the truncated LIA. This is because the truncation approach destroys the integrability of the underlying model (as we have shown in Sec. II B), with the result being that there are no longer an infinite number of conservation laws, and solutions such as solitons are no longer possible without such symmetry.

Another difference in the dynamics under a truncated LIA model could be seen in the structure of self-similar solutions. While self-similar solutions exist under the truncated LIA, the solutions must obey a $t^{1 / 3}$ similarity scaling in time, rather than the $t^{1 / 2}$ time scaling found under both classical LIA and BiotSavart dynamics. As this scaling is tied to how vortex filaments may radiate Kelvin waves to move energy between length scales after reconnection events (resulting in a smoothing of the vortex filament structure, particularly near the V-shaped kink which is formed), this suggests that the truncated LIA radiates Kelvin waves at a different (namely, slower) rate than does the classical LIA. This is sensible, as the development of the truncated LIA and the resulting local nonlinear equation was motivated by the need to allow more realistic Kelvin wave energy transfer, as the classical LIA is seemingly too simple to allow for this. ${ }^{15}$ Additionally, while the $t^{1 / 2}$ similarity scaling is more common in the literature, there is some support for $t^{1 / 3}$ type scalings. For instance, $t^{1 / 3}$ scalings appear to arise from reconnection events involving initially orthogonal vortex lines. ${ }^{60}$ This suggests that certain drastic departures from the integrable dynamics under the classical LIA are possible when considering the non-integrable truncated LIA, and hence the further study of the dynamics under models such as the local nonlinear equation-particularly in the case of multiple interacting filaments rather than isolated filaments considered here-may be of interest.

${ }^{1}$ S. K. Nemirovskii, "Quantum turbulence: Theoretical and numerical problems," Phys. Rep. 524, 85-202 (2013).

${ }^{2}$ B. V. Svistunov, "Superfluid turbulence in the low-temperature limit," Phys. Rev. B 52, 3647 (1995).

${ }^{3}$ M. D. Bustamante and S. Nazarenko, "Derivation of the Biot-Savart equation from the nonlinear Schrödinger equation," Phys. Rev. E 92, 053019 (2015).

${ }^{4}$ L. S. Da Rios, "Sul moto d'un liquido indefinito con un filetto vorticoso di forma qualunque (on the motion of an unbounded liquid with a vortex filament of any shape)," Rend. Circolo Mat. Palermo 22, 117-135 (1906).

${ }^{5}$ R. J. Arms and F. R. Hama, "Localized-induction concept on a curved vortex and motion of an elliptic vortex ring," Phys. Fluids 8, 553-559 (1965).

${ }^{6}$ H. Hasimoto, “A soliton on a vortex filament," J. Fluid Mech. 51, 477-485 (1972).

${ }^{7}$ D. Levi, A. Sym, and S. Wojciechowski, "N-solitons on a vortex filament," Phys. Lett. A 94, 408-411 (1983).
${ }^{8}$ M. Umeki, "A locally induced homoclinic motion of a vortex filament," Theor. Comput. Fluid Dyn. 24, 383-387 (2010).

${ }^{9}$ H. Salman, "Breathers on quantized superfluid vortices," Phys. Rev. Lett. 111, 165301 (2013).

${ }^{10}$ H. Salman, "Multiple breathers on a vortex filament," J. Phys.: Conf. Ser. 544, 012005 (2014).

${ }^{11}$ R. L. Ricca, "Torus knots and polynomial invariants for a class of soliton equations," Chaos 3, 83-91 (1993).

${ }^{12}$ E. J. Hopfinger and F. K. Browand, "Vortex solitary waves in a rotating, turbulent flow," Nature 295, 393-395 (1982).

${ }^{13}$ E. J. Hopfinger, F. K. Browand, and Y. Gagne, "Turbulence and waves in a rotating tank," J. Fluid Mech. 125, 505-534 (1982).

${ }^{14}$ T. Maxworthy, E. J. Hopfinger, and L. G. Redekopp, "Wave motions on vortex cores," J. Fluid Mech. 151, 141-165 (1985).

${ }^{15}$ J. Laurie, V. S. L'vov, S. Nazarenko, and O. Rudenko, "Interaction of Kelvin waves and nonlocality of energy transfer in superfluids," Phys. Rev. B 81, 104526 (2010).

${ }^{16}$ G. Boffetta, A. Celani, D. Dezzani, J. Laurie, and S. Nazarenko, "Modeling Kelvin wave cascades in superfluid helium," J. Low Temp. Phys. 156, 193-214 (2009)

${ }^{17}$ R. A. Van Gorder, "Translation of waves along quantum vortex filaments in the low-temperature two-dimensional local induction approximation," Phys. Fluids 27, 095104 (2015).

${ }^{18}$ R. A. Van Gorder, "Solitons and nonlinear waves along quantum vortex filaments under the low-temperature two-dimensional local induction approximation," Phys. Rev. E 93, 052208 (2016).

${ }^{19}$ S. E. Widnall, "The stability of a helical vortex filament," J. Fluid Mech. 54, 641-663 (1972).

${ }^{20}$ D. W. Moore and P. G. Saffman, "The motion of a vortex filament with axial flow," Philos. Trans. R. Soc., A 272, 403-429 (1972).

${ }^{21}$ R. L. Ricca, "The effect of torsion on the motion of a helical vortex filament," J. Fluid Mech. 273, 241-259 (1994).

${ }^{22}$ J. Boersma and D. H. Wood, "On the self-induced motion of a helical vortex," J. Fluid Mech. 384, 263-279 (1999).

${ }^{23} \mathrm{H}$. Zhou, "On the motion of slender vortex filaments," Phys. Fluids 9, 970-981 (1997).

${ }^{24}$ S. Kida, “A vortex filament moving without change of form," J. Fluid Mech. 112, 397-409 (1981).

${ }^{25}$ E. B. Sonin, "Dynamics of helical vortices and helical-vortex rings," Europhys. Lett. 97, 46002 (2012).

${ }^{26} \mathrm{R}$. A. Van Gorder, "Helical vortex filament motion under the non-local Biot-Savart model," J. Fluid Mech. 762, 141-155 (2015).

${ }^{27}$ A. Ramani, B. Grammaticos, and T. Bountis, "The Painlevé property and singularity analysis of integrable and non-integrable systems," Phys. Rep. 180, 159-245 (1989).

${ }^{28}$ J. Weiss, M. Tabor, and G. Carnevale, "The Painlevé property for partial differential equations," J. Math. Phys. 24, 522-526 (1983).

${ }^{29}$ J. Weiss, "On classes of integrable systems and the Painlevé property," J. Math. Phys. 25, 13-24 (1984).

${ }^{30}$ M. Russo, R. A. Van Gorder, and S. R. Choudhury, "Painlevé property and exact solutions for a nonlinear wave equation with generalized power-law nonlinearities," Commun. Nonlinear Sci. Numer. Simul. 18, 1623-1634 (2013).

${ }^{31}$ J. Hearns, R. A. Van Gorder, and S. R. Choudhury, "Painlevé test, integrability, and exact solutions for density-dependent reaction-diffusion equations with polynomial reaction functions," Appl. Math. Comput. 219, 3055-3064 (2012).

${ }^{32}$ R. L. Ricca, "The contributions of Da Rios and Levi-Civita to asymptotic potential theory and vortex filament dynamics," Fluid Dyn. Res. 18, 245-268 (1996).

${ }^{33} \mathrm{H}$. Hasimoto, "Motion of a vortex filament and its relation to elastica," J. Phys. Soc. Jpn. 31, 293-294 (1971).

${ }^{34}$ R. A. Van Gorder, "Integrable stationary solution for the fully nonlinear local induction equation describing the motion of a vortex filament," Theor. Comput. Fluid Dyn. 26, 591-594 (2012).

${ }^{35}$ R. A. Van Gorder, "Scaling laws and accurate small-amplitude stationary solution for the motion of a planar vortex filament in the Cartesian form of the local induction approximation,” Phys. Rev. E 87, 043203 (2013).

${ }^{36}$ S. Kida, "Stability of a steady vortex filament," J. Phys. Soc. Jpn. 51, 1655-1662 (1982).

${ }^{37}$ R. A. Van Gorder, "Orbital stability for rotating planar vortex filaments in the Cartesian and arclength forms of the local induction approximation," J. Phys. Soc. Jpn. 82, 094005 (2013). 
${ }^{38}$ Y. Fukumoto, "Stationary configurations of a vortex filament in background flows," Proc. R. Soc. A 453, 1205-1232 (1997).

${ }^{39}$ R. A. Van Gorder, "Exact solution for the self-induced motion of a vortex filament in the arc-length representation of the local induction approximation," Phys. Rev. E 86, 057301 (2012).

${ }^{40}$ R. A. Van Gorder, "Non-local dynamics governing the self-induced motion of a planar vortex filament," Phys. Fluids 27, 065105 (2015).

${ }^{41}$ R. A. Van Gorder, "General rotating quantum vortex filaments in the lowtemperature Svistunov model of the local induction approximation," Phys. Fluids 26, 065105 (2014).

${ }^{42}$ G. P. Bewley, M. S. Paoletti, K. R. Sreenivasan, and D. P. Lathrop, "Characterization of reconnecting vortices in superfluid helium," Proc. Natl. Acad. Sci. U. S. A. 105, 13707-13710 (2008).

${ }^{43}$ T. Lipniacki, "Evolution of quantum vortices following reconnection," Eur. J. Mech.-B/Fluids 19, 361-378 (2000).

${ }^{44}$ T. Lipniacki, "Quasi-static solutions for quantum vortex motion under the localized induction approximation," J. Fluid Mech. 477, 321-337 (2003).

${ }^{45}$ T. Lipniacki, "Shape-preserving solutions for quantum vortex motion under localized induction approximation," Phys. Fluids 15, 1381-1395 (2003).

${ }^{46}$ R. A. Van Gorder, "Self-similar vortex dynamics in superfluid 4He under the Cartesian representation of the Hall-Vinen model including superfluid friction," Phys. Fluids 25, 095105 (2013).

${ }^{47}$ S. Gutiérrez, J. Rivas, and L. Vega, "Formation of singularities and self-similar vortex motion under the localized induction approximation," Commun. Partial Differ. Equations 28, 927-968 (2003).

${ }^{48}$ R. B. Pelz, "Locally self-similar, finite-time collapse in a high-symmetry vortex filament model,” Phys. Rev. E 55, 1617 (1997).
${ }^{49}$ V. M. Fernandez, N. J. Zabusky, and V. M. Gryanik, "Vortex intensification and collapse of the Lissajous-elliptic ring: Single-and multi-filament BiotSavart simulations and visiometrics,” J. Fluid Mech. 299, 289-331 (1995).

${ }^{50}$ C. Das, S. Kida, and S. Goto, "Overall self-similar decay of two-dimensional turbulence,” J. Phys. Soc. Jpn. 70, 966-976 (2001).

${ }^{51}$ J. C. del Álamo, J. Jimenez, P. Zandonade, and R. D. Moser, "Self-similar vortex clusters in the turbulent logarithmic region," J. Fluid Mech. 561, 329-358 (2006)

${ }^{52}$ Y. Kimura, "Similarity solution of two-dimensional point vortices," J. Phys. Soc. Jpn. 56, 2024-2030 (1987).

${ }^{53}$ Y. Kimura, "Self-similar collapse of a 3D straight vortex filament model," Geophys. Astrophys. Fluid Dyn. 103, 135-142 (2009).

${ }^{54}$ Y. Kimura, "Self-similar collapse of 2D and 3D vortex filament models," Theor. Comput. Fluid Dyn. 24, 389-394 (2010).

${ }^{55} \mathrm{H}$. Yoshimoto and S. Goto, "Self-similar clustering of inertial particles in homogeneous turbulence," J. Fluid Mech. 577, 275-286 (2007).

${ }^{56}$ Y. Kimura and K. Moffatt, "Scaling properties towards vortex reconnection under Biot-Savart evolution," Fluid Dyn. Res. (in press).

${ }^{57}$ R. A. Van Gorder, "Self-similar vortex filament motion under the non-local Biot-Savart model," J. Fluid Mech. 802, 760-774 (2016).

${ }^{58}$ E. Fonda, D. P. Meichle, N. T. Ouellette, S. Hormoz, and D. P. Lathrop, "Direct observation of Kelvin waves excited by quantized vortex reconnection,” Proc. Natl. Acad. Sci. U. S. A. 111, 4707-4710 (2014).

${ }^{59}$ A. Villois, D. Proment, and G. Krstulovic, "Universal and nonuniversal aspects of vortex reconnections in superfluids," Phys. Rev. Fluids 2044701 , (2017).

${ }^{60}$ C. Rorai, J. Skipper, R. M. Kerr, and K. R. Sreenivasan, "Approach and separation of quantised vortices with balanced cores," J. Fluid Mech. 808, 641-667 (2016). 